\title{
Situación demográfica de la infancia y la adolescencia actual. Una visión mundial $^{1}$
}

\author{
Alberto Sanz Gimeno ${ }^{2}$ \\ Recibido: 21 de febrero de 2020 / Aceptado: 19 de junio de 2020
}

Resumen. Este trabajo recoge datos e indicadores de carácter demográfico sobre los niños y los adolescentes a partir de las principales estadísticas producidas por organismos internacionales. El objetivo que aborda es doble. Por un lado, hacer un retrato a nivel mundial de la situación de la infancia y la adolescencia actual, destacando aquellos aspectos que, siempre desde una óptica demográfica, requieren una especial atención pues conforman procesos sociales que afectan de forma sustantiva a su modo y calidad de vida. Y por otro, mostrar a los investigadores de otros campos disciplinares, que los datos e indicadores demográficos sirven no solo para contextualizar algunos de estos procesos, sino también, para identificarlos y analizarlos, procurando un conocimiento útil de cara a las colaboraciones interdisciplinares que, muchas veces, son necesarias para la elaboración de propuestas de acción eficaces.

Palabras clave: Demografía de la infancia y de la adolescencia; estructura de la población; hogar; familia; nupcialidad adolescente; fecundidad adolescente; mortalidad de la infancia y de la adolescencia; causas de muerte; migraciones de niños y jóvenes.

\section{[pt] Situação demográfica da infância e adolescência hoje. Uma visão do mundo}

Resumo. Este trabalho coleta dados demográficos e indicadores sobre crianças e adolescentes a partir das principais estatísticas produzidas por organizações internacionais. O objetivo que ele aborda é duplo. Por um lado, para fazer um retrato mundial da situação atual da infância e adolescência, destacando os aspectos que, sempre do ponto de vista demográfico, requerem atenção especial, pois formam processos sociais que afetam substancialmente seu modo e qualidade de vida. Por outro lado, mostrar a pesquisadores de outras áreas disciplinares que dados e indicadores demográficos servem não apenas para contextualizar alguns desses processos, mas também para identificá-los e analisá-los, fornecendo conhecimento útil para colaborações interdisciplinares que, muitas vezes, são necessárias para a elaboração de propostas de ação eficazes.

Palavras chave: Demografia da infância e adolescência; estrutura populacional; casa; família; nupcialidade do adolescente; fertilidade do adolescente; mortalidade na infância e adolescência; causas de morte; migração de crianças e jovens.

\section{[en] Demographic Situation of Today’s Childhood and Adolescence. A World View}

Abstract. This work collects demographic data and indicators on children and adolescents from the main statistics produced by international organizations. There is a double objective. On the one hand, make a worldwide portrait of the current situation of childhood and adolescence, highlighting those aspects that (from a demographic perspective), require special attention because they form social processes that affect their mode and quality of life. And on the other, to show researchers from other disciplinary fields that demographic data and indicators serve to contextualize some of these processes, but also, to identify and analyze them, providing useful knowledge for interdisciplinary collaborations. Many times, these collaborations are necessary for the elaboration of effective action proposals.

Keywords: Demography of childhood and adolescence; population structure; home; family; adolescent nuptiality; adolescent fertility; infant and childhood mortality; adolescence mortality; causes of death, migration of children and young people.

Sumario. 1. Introducción. 2. Volumen y estructura de la infancia y adolescencia a nivel mundial. 2.1. Diferencias de edad. 2.2. Diferencias por sexo. 3. Características de los hogares de niños y adolescentes. 4. La nupcialidad adolescente. 5.

\footnotetext{
Este trabajo se ha realizado en el marco del programa de actividades del grupo de investigación GEPS (Grupo de Estudios “Población y Sociedad"), financiado por la Comunidad de Madrid (H2019/HUM-5802 - GEPS-CM).

2 Profesor titular en la Universidad Complutense de Madrid.

E-mail: alsanz@ucm.es 
La fecundidad adolescente. 6. La mortalidad y sus causas en la infancia y la adolescencia. 7. El papel de los niños y los adolescentes en las migraciones internacionales. 8. Conclusiones. 9. Referencias bibliográficas.

Cómo citar: Sanz Gimeno, A. (2020): Situación demográfica de la infancia y la adolescencia actual. Una visión mundial, Sociedad e Infancias, 4, 5-21.

\section{Introducción}

El 20 de noviembre de 1989 se firmaba la Convención sobre los Derechos del Niño. En aquel año, la población del planeta superaba los 5200 millones de habitantes y los niños y los adolescentes (habitualmente comprendidos entre 0 y 17 años), con unos 2000 millones, suponían un 39\% de todas las personas existentes. Treinta años después, en 2019, y pese a haber superado las etapas de mayor aceleración del crecimiento demográfico correspondientes a las décadas de los años sesenta y setenta del siglo XX (Arango Vila-Belda 2004), la población global ha aumentado hasta algo más de 7700 millones. Sin embargo, los niños y los adolescentes, pese a ser más en cifras absolutas (unos 2342 millones), han perdido peso relativo y suponen un $30.3 \%$ de la población mundial actual.

Para aquellos que piensen que esta reducción no es importante, es conveniente aclarar que se trata de una disminución del $21 \%$, es decir, un poco más de una quinta parte del peso relativo que la infancia y la adolescencia tienen en la población general y este peso no es nada desdeñable. Mientras que muchos países se encuentran aún en ese proceso de cambio que se conoce como Transición Demográfica (Notestein 1945; Chesnais 1992), buena parte de la población, sobre todo en los países con cierto nivel de desarrollo, se enfrentan a un reto importante protagonizado por el fenómeno del envejecimiento demográfico. En muchos de estas sociedades, consideradas como las más modernas, el aumento de la longevidad (sustentado en una mejora de la salud y de la esperanza de vida) y la reducción de la fecundidad han conducido a un cambio de sus estructuras demográficas, en las que cada vez los individuos de más edad tienen un peso relativo mayor. Sin embargo, salvo una extensa preocupación por cómo hacer para remontar los bajos niveles de fecundidad en algunos países desarrollados (habitualmente por debajo de 2.1 y de 1.5 hijos por mujer), no abundan en demasía los trabajos orientados hacia las características sociodemográficas del colectivo más joven de la población y, en todo caso, su existencia es más común cuando se trata de analizar situaciones de pobreza, marginalidad y vulnerabilidad que, lamentablemente, aún persisten entre la infancia y adolescencia de muchas poblaciones. Merece la pena destacar, en este sentido, los informes de UNICEF sobre "El Estado Mundial de la Infancia", que suelen incluir datos sobre niños y adolescentes en distintos ámbitos de su vida.

El presente trabajo trata de dar algún paso en esa necesidad de ahondar más en las características de este grupo de población, centrando su atención sobre una visión demográfica general acerca de cómo se encuentran los niños y los adolescentes en el mundo actual y cuáles son algunos de los fenómenos que deben ser objeto de atención para la agenda investigadora de hoy y de los próximos años. En este sentido, este documento tiene un carácter eminentemente prospectivo y descriptivo. Se trata de poner de manifiesto la capacidad de diversos indicadores demográficos para hacer un buen retrato de la población de 0 a 17 años en el mundo, de presentar su relación con otras dinámicas demográficas y sociales y de apuntar, también, algunos temas sobre los que la investigación debería centrarse y detallarse para conseguir un buen diagnóstico de situaciones que requieren especial atención e intervenciones concretas.

Un trabajo de estas características difícilmente puede permitirse un desarrollo detallado sobre las cuestiones abordadas. Probablemente, ahí está su mayor deuda o debilidad, pero sí permite proporcionar al lector alguna información básica (puede que hasta útil), sobre qué aspectos pueden ser relevantes y cómo se puede llegar a una lectura adecuada de los mismos. La idea es esta, presentar la capacidad de los indicadores demográficos para abordar algunos temas de interés en torno a la infancia y la adolescencia, visibilizar aspectos específicos de este grupo de la población y presentar un estado general de la situación actual del mismo desde una perspectiva global.

Los datos e indicadores en los que vamos a basarnos han sido producidos por organismos internacionales de reconocida solvencia, si bien ello no implica que no existan ciertas inexactitudes y carencias. La fuente más usada para este trabajo ha sido el World Population Prospects, elaborado por Naciones Unidas en su edición para el año 2019. Se trata de una fuente de indudable interés que pone a disposición de los investigadores estimaciones de los principales datos e indicadores demográficos desde 1950 hasta la actualidad, incluyendo, también, una proyección de los mismos. La desventaja de esta fuente radica, quizás, en que presenta datos agrupados por quinquenios (solo hay algunas estadísticas con detalle anual). Se trata, en buena medida, de estimaciones realizadas por la División de Población de Naciones Unidas a partir de los datos proporcionados por los institutos de estadística de cada país, o en su defecto, partiendo de encuestas realizadas por Naciones Unidas y otros organismos colaboradores. Dichas encuestas suelen tener un alto grado de fiabilidad, pero ello no quita la existencia de sesgos de cierto alcance.

Junto a esta gran fuente de información, se van a usar otros datos de carácter más concreto, que producen otros organismos como UNICEF. En este caso, la disponibilidad de información es algo menor ya que no hay datos para todos los países y regiones y hay ciertas carencias, pero al menos permiten identificar rasgos generales de la población analizada. 


\section{Volumen y estructura de la infancia y adolescencia a nivel internacional}

Como se mencionaba al iniciar este trabajo, una de las características más relevantes de la infancia y la adolescencia en el mundo actual es su evolución cuantitativa que, si bien revela un leve aumento en sus cifras brutas, tiene especial interés si consideramos sus cifras relativas, que son las habitualmente usadas en el análisis social y demográfico.

Gráfico 1. Porcentaje de población de 0 a 17 años en el mundo. Evolución y perspectivas, 1950-2100.

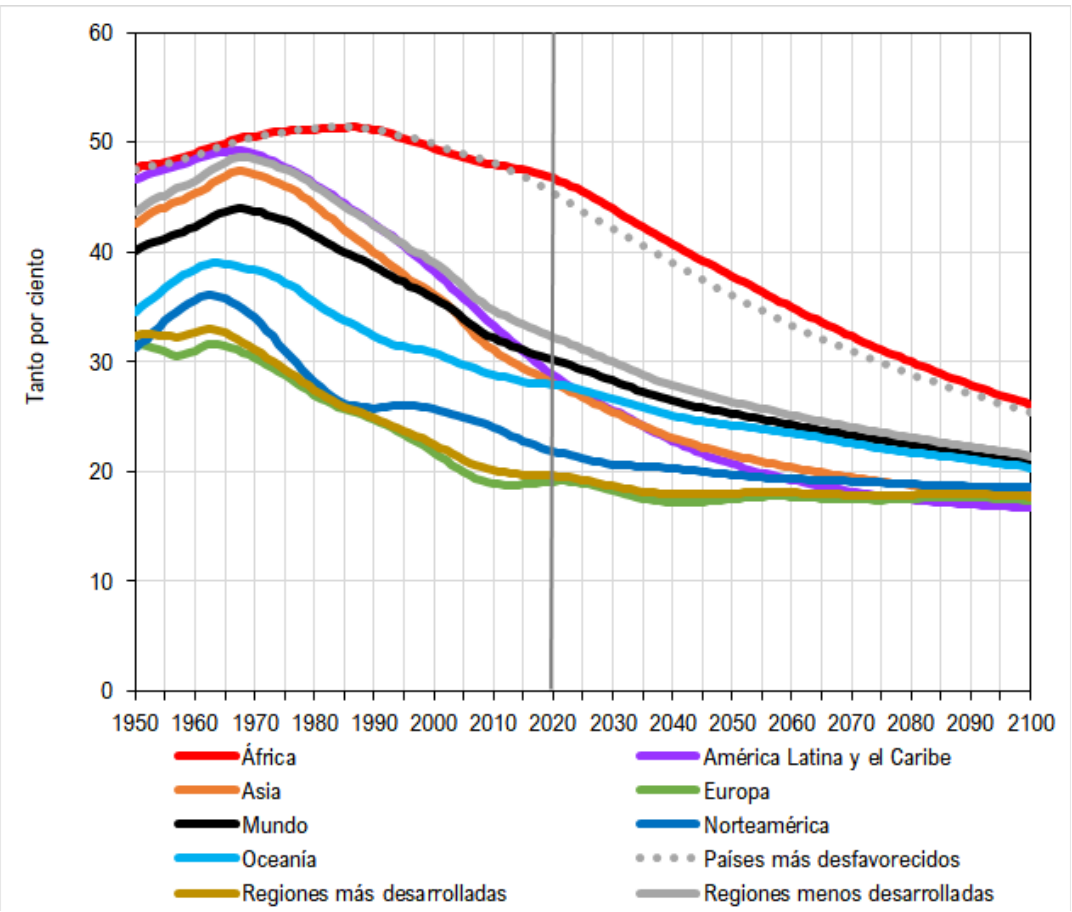

Elaboración propia a partir de los datos de: United Nations, Department of Economic and Social Affairs, Population Division (2019b). World Population Prospects 2019, (https://population.un.org/wpp/).

Nota: La línea gris vertical identifica el momento actual.

En este sentido, si fijamos nuestra atención en los datos disponibles para el mundo y para varias regiones del planeta, podemos apreciar la clara evolución descendente del peso de la población de 0 a 17 años en las últimas décadas (Gráfico 1).

El momento álgido, con un mayor peso de niños y jóvenes, se produjo en el quinquenio de 1965-70, cuando este grupo de población representaba, aproximadamente, un $44 \%$ de la población total del planeta. En aquella fecha, los altos índices de fecundidad en los años previos, incluidos los del denominado "baby boom" (van Bavel and Reher 2013), llevaron a una elevación sin precedentes en la historia de la humanidad del número de niños y adolescentes. A tan solo 6 puntos de suponer la mitad de la población de la Tierra ${ }^{3}$, este indicador da una idea del enorme potencial reproductivo que acompañó la denominada Transición Demográfica y de las consecuencias de que muchos países no siguieran al pie de la letra el dictamen de este modelo propio de la teoría demográfica. De hecho, de acuerdo con la Transición Demográfica, las poblaciones deberían haber ajustado claramente su fecundidad tras el declive anterior de la mortalidad. Sin embargo, muchos países en vías de desarrollo solo experimentaron la caída de la mortalidad, demorando durante mucho tiempo la esperada reducción de la fecundidad. Esto ha dado lugar a elevaciones importantes de la tasa de crecimiento demográfico y a la aparición de un enorme contingente de niños y jóvenes durante este período, que se conoce como el de "crecimiento acelerado de la población" (Arango Vila-Belda 2004; Livi-Bacci 2002).

Pese a la similitud de la tendencia global de estas dinámicas, puede apreciarse la existencia de importantes diferencias entre los países más desarrollados y los menos desarrollados ${ }^{4}$, fruto de la distinta cronología con que la transición demográfica se ha producido en unos y otros (Reher, 2004). El mundo más desarrollado, básicamente países de Europa y Norteamérica (junto a otros como Japón o Australia) estaba mostrando, ya a mediados de los años sesenta, el mismo peso relativo que los niños y los adolescentes tienen a nivel mundial en la actualidad. El resto de las regiones: Asia, Oceanía y América Latina han tardado unos 50 años en experimentar la situación

\footnotetext{
En el caso del continente africano, al igual que en el grupo de países que conforman las "poblaciones más desfavorecidas" del planeta, se superó claramente el umbral del $50 \%$.

4 Para este trabajo se han tomado las agrupaciones de países que realiza Naciones Unidas con relación al nivel de desarrollo de los mismos (United Nations - Department of Economic and Social Affairs 2019).
} 
propia de los países europeos. El continente africano, que muestra claramente un gran retraso en esta evolución y es el único que hoy mantiene una elevada proporción de niños y jóvenes, muestra una tendencia descendente, que se inicia con mayor retraso y que tiene que esperar a la última década del siglo XX y primeros años del XXI para comenzar a consolidarse.

El futuro, si es que se cumplen las previsiones hechas por los organismos internacionales bajo el auspicio de un "pretendido equilibrio demográfico", revela una clara fijación de este bajo peso relativo de los niños y los adolescentes en el mundo desarrollado y una tendencia de disminución en el resto de las regiones. Así, la infancia y la adolescencia llegarían a suponer para el año 2100 solo una quinta parte de la población mundial.

De este modo, la evolución demográfica refleja cierta paradoja y es que, mientras se incrementa la atención y la protección sobre la situación y derechos de la infancia y la adolescencia, el peso de este grupo de población pierde entidad progresivamente, dándose una situación de hecho en la que las mayores debilidades y vulnerabilidades de los niños y adolescentes se dan, precisamente, en aquellos lugares del mundo donde su peso en la población es más elevado.

Mapas 1 y 2. 1)Porcentaje de población de 0 a 17 años en el mundo. Años 2019. 2)Reducción (\%) de la población de 0 a 17 años en el mundo. Desde la fecha con el valor máximo hasta el año 2019.
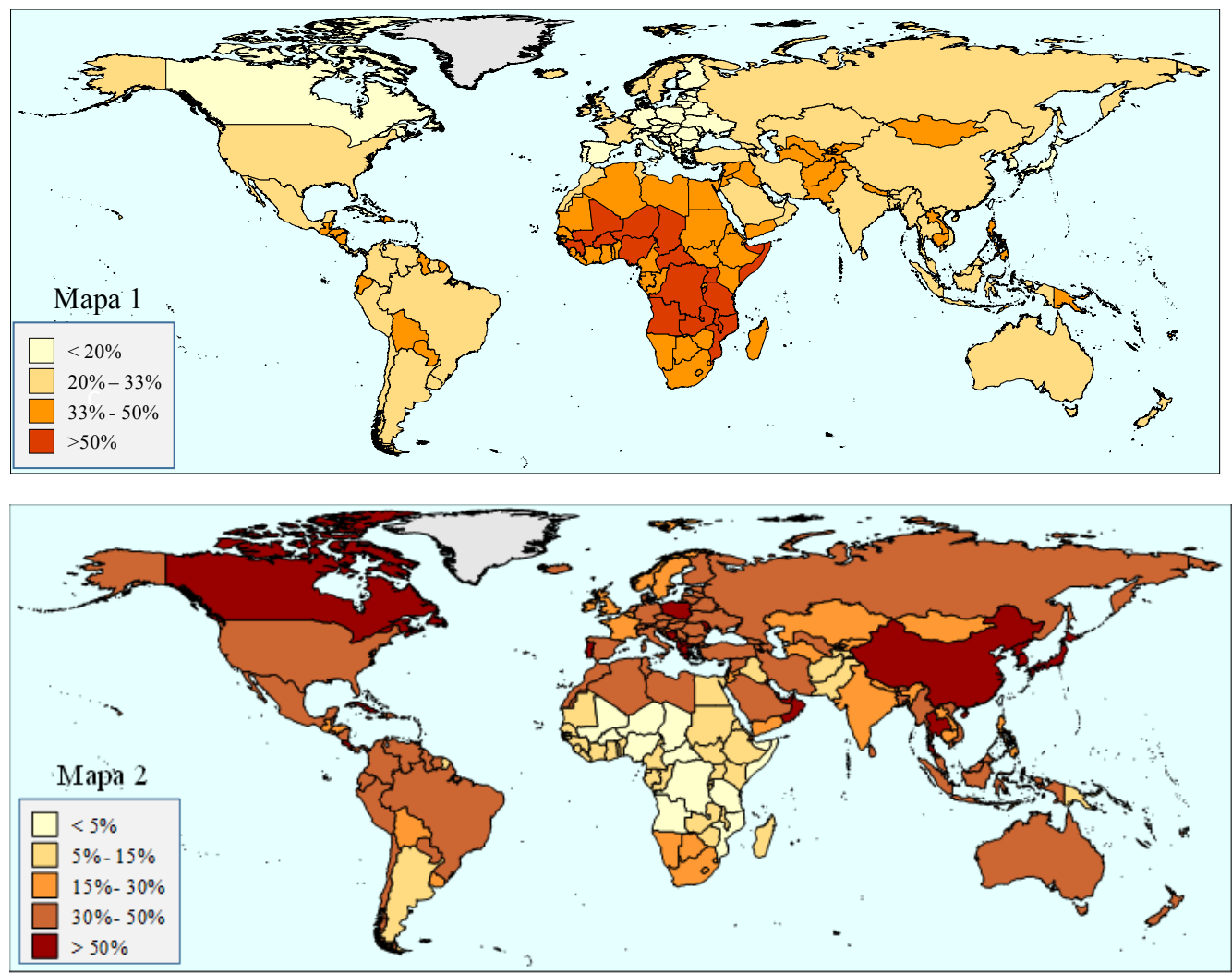

Elaboración propia a partir de los datos de: United Nations, Department of Economic and Social Affairs, Population Division (2019b). World Population Prospects 2019, (https://population.un.org/wpp/).

En los mapas 1 y 2, hemos tratado de representar este proceso de cambio teniendo en cuenta un mayor nivel de detalle (por países). El mapa 1 nos ofrece el peso de la infancia y adolescencia en la población total actual, siendo claramente apreciable como el continente africano, algunos países de Asia Occidental y Meridional y, también, aunque en un menor nivel, algunos pases de América Latina son actualmente las zonas que presentan una importante representación de este grupo de población, cuya situación tiene claramente que ver con la evolución mostrada en el mapa 2. Este último recoge el nivel relativo de reducción del peso de este grupo de población desde el año en que cada país ha mostrado su valor más alto hasta la actualidad. Ciertamente, contiene un sesgo importante respecto al hecho de que cada país tiene su propia cronología en esta transformación, pero permite una clara visualización de la intensidad de los cambios. Así, China, Canadá y algunos países, sobre todo de la Europa Oriental, se destacan por una gran pérdida de peso de la infancia y la adolescencia. En el caso chino, esta situación responde a los efectos de la aplicación durante varias décadas de la política del hijo único. En otros de los países representados, la reducción tiene que ver con la instauración de un régimen de baja fecundidad, tal y como preconizan los marcos teóricos de la Transición Demográfica y de la Segunda Transición Demográfica. Esta última señala un aumento del individualismo, la revolución de las concepciones sexuales y procreativas, la mayor igualdad de las mujeres y el aumento en el uso 
de medios anticonceptivos eficaces, entre otros factores determinantes de esta baja fecundidad (Lesthaeghe and Van de Kaa, 1986).

Es nuevamente palpable la escasa transformación experimentada por buena parte de los países del continente africano donde, salvo los países ribereños del norte y los de la punta sur, el resto muestra que los niños y los adolescentes constituyen, aproximadamente, la mitad de la población en muchos de ellos. Así, los hijos no solo constituyen un importante activo cultural claramente relacionado con el valor procreativo de los individuos y de las familias, sino que, también, suponen un recurso económico, colaborando con su trabajo para lograr el sostén y el bienestar de los miembros de la familia, del clan o de la tribu. Mientras determinados valores culturales y económicos predominen frente a otras influencias externas será complicado apreciar cambios ostensibles en los niveles reproductivos de muchas parejas africanas.

\subsection{Diferencias por edad}

Cabe preguntarse si esta evolución general afecta de manera homogénea a toda la infancia y la adolescencia o si se pueden apreciar diferencias en función de otras variables demográficas como la edad y el sexo.

No hay una clara convención acerca de las edades que delimitan cada una de las etapas vitales del desarrollo del ser humano y esto atañe, también, a la infancia y a la adolescencia. Cada uno de estos períodos tiene una duración variable, que depende en muchos casos de las circunstancias sociales, culturales y de desarrollo económico de cada sociedad (por ejemplo, por el efecto de las condiciones de salud y nutrición de los individuos) y, también, de otros aspectos de carácter personal, tanto en el ámbito del desarrollo fisiológico como en el mental y psicológico. Desde luego, las estadísticas internacionales no hacen alusión a edades específicas desde un criterio homologado, sino que, por lo general, suelen distinguir entre la infancia (considerando habitualmente desde el nacimiento hasta los 5 años de edad), y la adolescencia (habitualmente entre los 10 y los 17 años de edad).

Por ello, en nuestra búsqueda de datos, no hemos podido conseguir siempre información concreta para este conjunto de la población de manera claramente diferenciada y, en el mejor de los casos, hemos podido hacer, de manera arbitraria, nuestras propias agrupaciones. Esto nos ha permitido obtener cierto grado de detalle para responder a esa necesidad de apreciar si hay comportamientos homogéneos o heterogéneos entre niños y adolescentes.

En el gráfico 2, hemos representado la evolución del peso de los niños y los adolescentes en grandes regiones del mundo, distinguiendo 3 grandes grupos de edad, que tienen la virtud de tener una misma amplitud (6 años) y, en buena medida, de representar 3 etapas relevantes de la vida de cada individuo: la primera infancia (entre 0 y 5 años), la etapa escolar y preadolescente (entre 6 y 11 años) y la adolescencia (entre 12 y 17 años). Cada una de estas etapas podría caracterizarse por rasgos o elementos comunes inherentes a los acontecimientos que las sociedades suelen deparar para las personas en estas edades. Desafortunadamente, y por el hecho de que estamos ante una revisión mundial, resulta bastante difícil hacer equiparables o diferenciables las condiciones que acompañan a estos grupos de edad en unas sociedades u otras. No obstante, no renunciamos a la relevancia de contemplar la evolución demográfica del peso de la población en estos tres grupos de edad.

Así, se hace evidente que, en el mundo (primer gráfico a la izquierda), el peso de la primera infancia ( 0 a 5 años) ha sido claramente mayor desde 1950 hasta prácticamente la actualidad. Este grupo ha pasado de representar un $17 \%$ de la población mundial a situarse en torno a un $11 \%$ hoy en día. En segundo lugar, el grupo de edad de mayor peso es de los niños en edad escolar-preadolescente ( 6 a 11 años) que, si bien llegaron a suponer un 15\% en los años sesenta del siglo XX, hoy han descendido en su peso hasta un valor del 10\%. Por último, los adolescentes (12 a 17 años) han tenido un peso algo menor en su punto álgido, poco más de un $12 \%$ y en la actualidad ni siquiera llegan al $10 \%$ antes citado. Lo más relevante de esta evolución es que si en el pasado se trataba de 3 grupos de edad claramente diferenciados en su peso, en la actualidad tienden claramente a una convergencia numérica, que tiende a agudizarse en los próximos años en esa evolución de progresiva reducción de su peso que hemos comentado anteriormente. Las razones de esta tendencia tienen que ver con la distinta influencia que la fecundidad y los niveles de mortalidad han ejercido en sus cifras (y, en cierta medida, también por el efecto de las migraciones).

Este proceso de igualación en el peso que tienen estos grupos en el conjunto de la población es visible en todas las regiones analizadas, incluso en aquellas donde todavía faltan unos cuantos años para conseguir dicha convergencia, como pueden ser los países más desfavorecidos y, dentro de ellos, el caso de África. La razón de este tipo de evolución tiene que ver con la progresiva disminución de las diferencias en la fecundidad de las mujeres de unas cohortes reproductivas a otras y la reducción de los niveles de mortalidad a valores bajos y estables entre unas generaciones y otras. 
Gráfico 2. Porcentaje de población de 0 a 17 años en grandes grupos de edad respecto a la población total. Evolución y perspectivas regionales, 1950-2050.
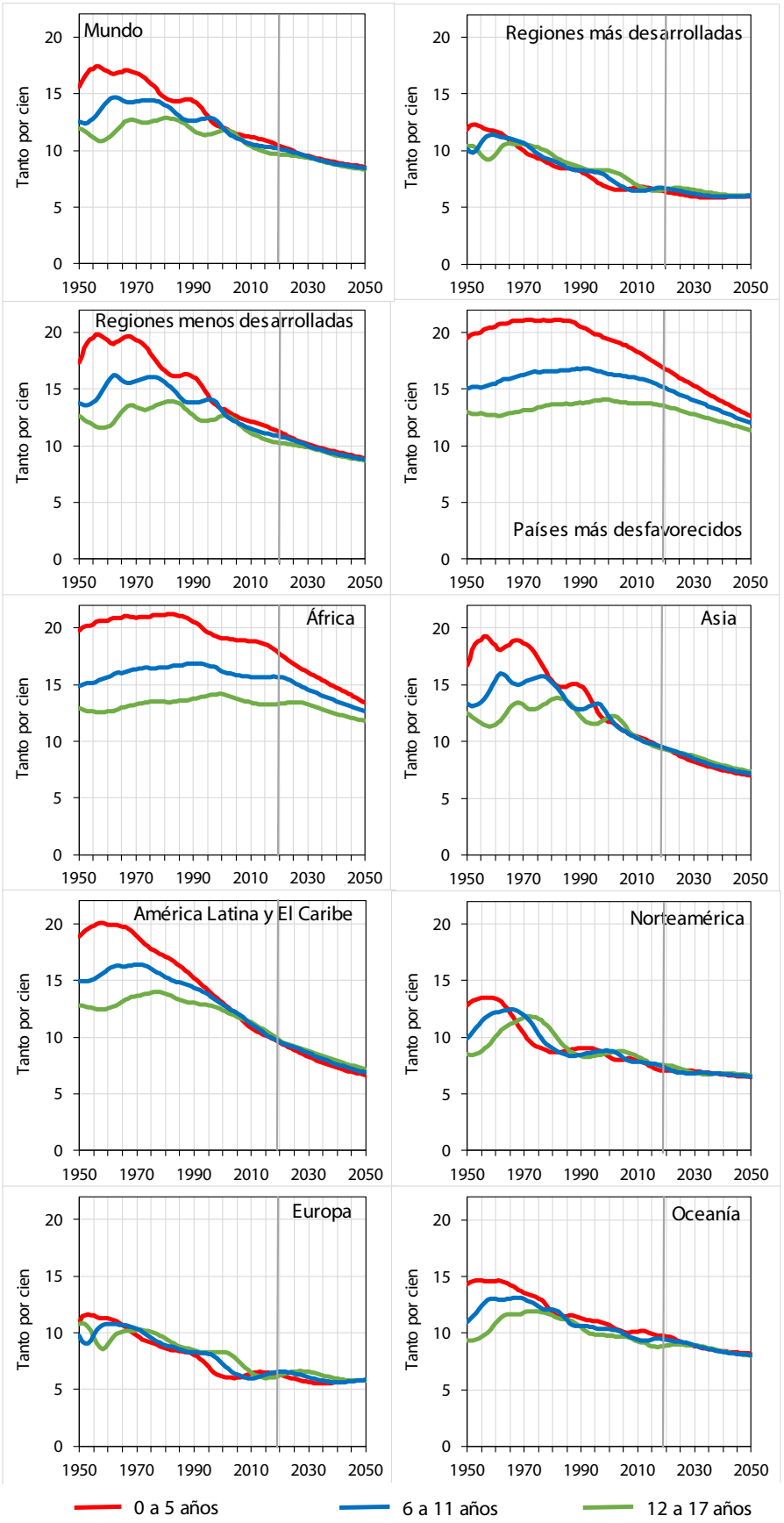

Elaboración propia a partir de los datos de: United Nations, Department of Economic and Social Affairs, Population Division (2019b). World Population Prospects 2019, (https://population.un.org/wpp/).

Será relevante tener en cuenta este tipo de proceso y sus características. Si la situación demográfica tiende a una clara equiparación estadística de estos grupos, todo lo contrario, tendrá que ser el desarrollo de la atención y los programas de acción en la infancia y la adolescencia. La disparidad de situaciones y necesidades que configuran estas etapas de los individuos precisarán, probablemente, de un tratamiento cada vez más pormenorizado, que tenga en cuenta los rasgos que identifican a los sujetos en cada uno de estos grupos de edad. Mientras los aspectos demográficos estructurales tenderán a hacerse menos visibles en algunas dimensiones, habrá que procurar la identificación y visualización de las distintas partes que incluyen la infancia y la adolescencia.

\subsection{Diferencias por sexo}

Junto a estos rasgos de los niños y los adolescentes según su edad, hay que prestar también especial atención a las diferencias por sexo. En demografía, el equilibrio entre los sexos suele constituir un elemento clave de las estructuras 
de cualquier población, pues ello supone la posibilidad de asegurar un reemplazo generacional en la medida que dicho equilibrio contribuye a la disponibilidad de pareja, al menos de cara al proceso reproductivo en dicha población. En la actualidad, sabemos que dicho equilibrio dista de ser perfecto y que hay etapas en la vida en la que distintas circunstancias favorecen la existencia de una proporción dispar de varones y mujeres. La mayor esperanza de vida de las mujeres es una de estas características que denota como las condiciones de existencia y de salud se configuran de forma diferente para unos y otras y explica porque el envejecimiento es protagonizado más por mujeres que por hombres, sobre todo a medida que avanzamos en la edad de los sujetos. Buena parte de los factores que explican estas disparidades son de carácter social, cultural y económico, pero hay también elementos de marcado origen biológico que nos ayudan a entender este rasgo en la estructura de cualquier población. Así, por ejemplo, la observación empírica ha puesto de manifiesto la existencia de cierto desequilibrio en la proporción de nacimientos masculinos y femeninos, de manera que es habitual encontrarse una razón entre los sexos de 106 niños por cada 100 niñas si solo actúan las fuerzas naturales sobre dicha población (Livi-Bacci, 1993).

Con este conocimiento en nuestra mano, no deja de ser sorprendente encontrar poblaciones que se alejan claramente de estos valores "esperables" para mostrar situaciones que pueden llevar a evidenciar, bien un problema en la calidad y fiabilidad de los datos analizados, bien una clara preferencia por un sexo con respecto al otro en el ámbito familiar y social. Algo así podemos contemplar en el mapa 3, que muestra la situación de los distintos países respecto a esta relación entre los sexos en el momento del nacimiento.

Si el color anaranjado muestra lo que podríamos llegar a considerar valores normales en esta razón, el color amarillo muestra una menor proporción de varones en el nacimiento con respecto a las cifras esperables. Esta situación, donde hay menos niños de los esperados o, incluso, mayor presencia de niñas parece ser muy clara en varios países del África Subsahariana y en algún país de Oriente Medio y de Asia. En ningún caso puede descartarse la influencia de algún problema en los datos. Sin embargo, cabe la posibilidad de que la mortalidad fetal de varones sea mayor que la de las mujeres ante una situación donde no se dan comportamientos selectivos en el acceso a la nutrición u otros componentes de la salud, por lo que finalmente podríamos encontrar un pequeño sesgo en la razón entre los sexos favorable a las mujeres (Svedberd, 1990).

Mapa 3. Razón entre los sexos al nacimiento en el mundo. Periodo 2015-2020.

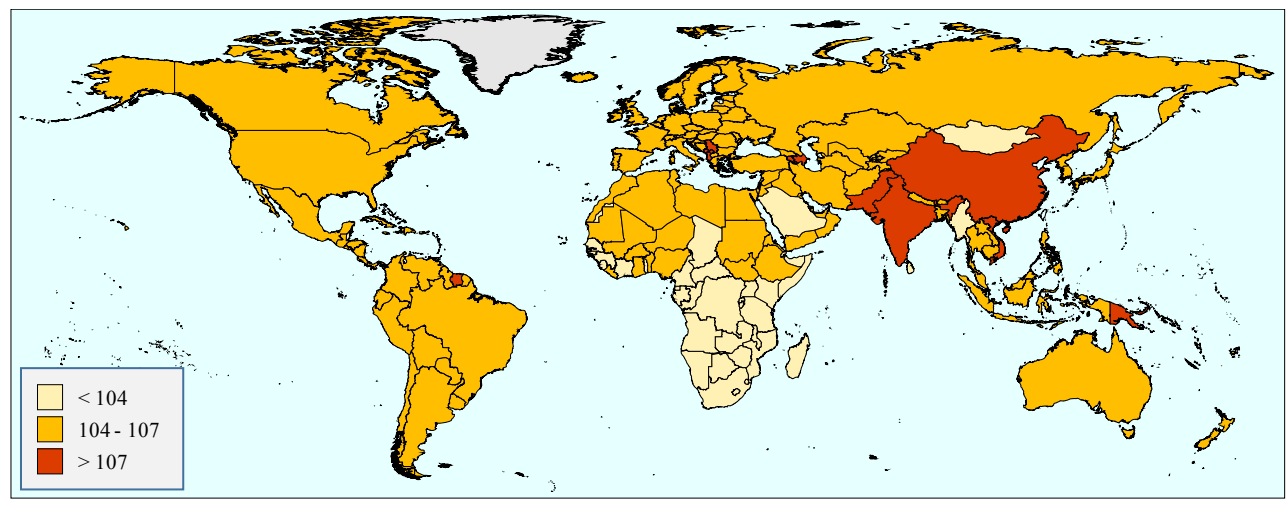

Elaboración propia a partir de los datos de: United Nations, Department of Economic and Social Affairs, Population Division (2019b). World Population Prospects 2019, (https://population.un.org/wpp/).

El fenómeno contrario: la preferencia clara por los varones (color rojo) es claramente visible en países como China, India y Pakistán, junto a otros de Asia. En varios de estos países, la adopción de prácticas discriminatorias por las que muchas parejas deciden practicar un aborto si el feto es femenino o, incluso, abandonan a la hija y/o prescinden del hecho registral si el nacimiento es de una niña, explican esta situación (Arnold and Zhaoxiang, 1986; Arnold et al., 2002; Arokiasamy, 2002; Attané, 2006; Attané, 2013; Das Gupta et al., 2002; Goodkind, 1996; Hesketh et al., 2011). Esta mayor preferencia por los varones no solo es visible en el momento del nacimiento, se manifiesta también en otras edades y no es extraño encontrar países donde la razón entre los sexos muestra un claro desequilibrio a favor de los varones en niños en la edad escolar y durante la adolescencia.

\section{Características de los hogares de niños y adolescentes}

Otro de los rasgos sociodemográficos fundamentales tiene que ver con las características del hogar y del grupo familiar o corresidencial. En este sentido, el hecho más destacable es que la reducción de la fecundidad, junto a otros factores asociados a la conformación del ciclo vital familiar (en el que hay que considerar las edades de emancipación, los procesos de emparejamiento y, también, de disolución de las parejas, entre otros), han venido propiciando una progresiva 
reducción del tamaño medio de los hogares, conformando un gradiente diferencial que distingue con claridad la composición y estructura de los hogares en los que viven los niños y los adolescentes en distintas partes del mundo.

Aunque la disponibilidad de datos en cuanto al número de países con información y la homogeneidad cronológica de las fechas para las que se aporta información dista de ser perfecta, sí es posible tener una panorámica general de cómo varía el tamaño medio del hogar a nivel mundial (gráfico 3).

Gráfico 3. Tamaño medio del hogar y porcentaje de hogares con población de 0 a 17 años en el mundo. Datos más recientes (desde 2000).

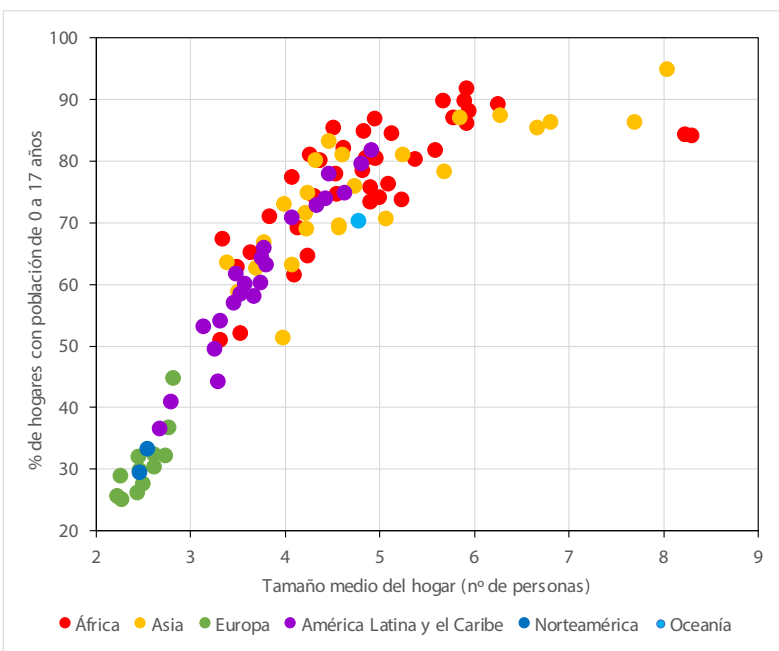

Elaboración propia a partir de los datos de: United Nations, Department of Economic and Social Affairs, Population Division (2018a). Database on Household Size and Composition 2018, (https://population.un.org/Household/index.html) y de United Nations (2017). Household Size and Composition Around the World 2017. Data booklet.

Considerando todos los países con información, el tamaño medio del hogar en el mundo se sitúa en la actualidad en 3.6 miembros. Esto quiere decir, haciendo una deducción rápida de situaciones estándar, que lo habitual son la pareja progenitora con 1-2 hijos, siendo importante la proporción de hogares que no llegan a tener esos dos hijos. La situación varía desde continentes como África, que tienen 4.8 miembros y Asia con 4.3, al caso de Europa o Norteamérica con hogares de 2.5 miembros como media.

En el gráfico 3 se aprecia, también, la estrecha relación existente entre el tamaño medio del hogar con el número de niños y adolescentes presente en el mismo. Los puntos que representan a África y Asia se mantienen en los valores elevados de ambos ejes; América Latina y el Caribe en una posición intermedia (3.6 miembros como tamaño medio) y los valores más bajos aparecen en Norteamérica y Europa.

Gráfico 4. Características (\%) de los hogares con niños y adolescentes en el mundo. Datos más recientes (desde 2000).

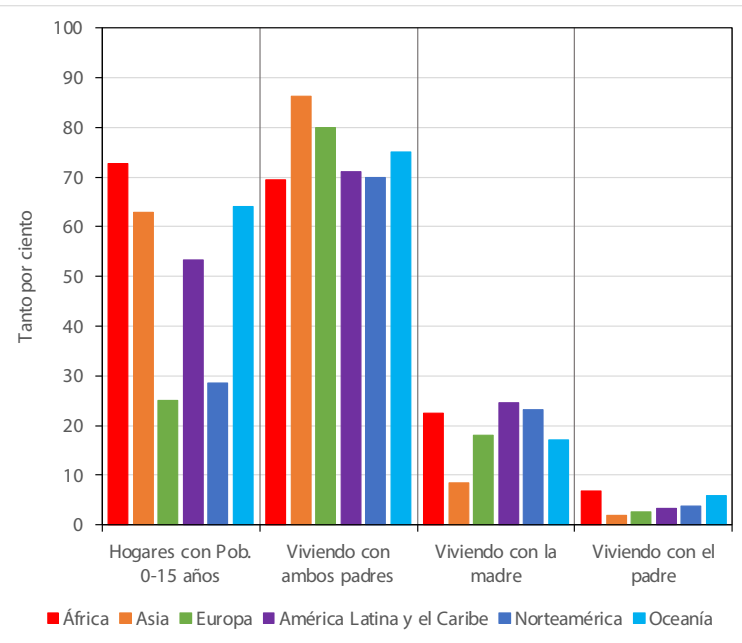

Elaboración propia a partir de los datos de: United Nations (2017). Household Size and Composition Around the World 2017. Data booklet. Nota: Los valores son la media ponderada de cada indicador considerando la población de los respectivos países en cada región. 
Respecto al estado de corresidencia, como hemos mencionado, no abundan las estadísticas con grandes detalles, pero como se puede apreciar en el gráfico 4, la situación más habitual, pues supone un $70 \%$ o más de los hogares con niños y adolescentes en el mundo, es la de convivir con ambos padres, siendo este modelo de convivencia mayoritario en Asia y en Europa ${ }^{5}$. Ahora bien, a la hora de precisar las posibles preferencias o situaciones habituales ante el crecimiento de los hogares monoparentales (relacionado con los procesos de disolución de las parejas en mayor medida que por la mortalidad de alguno de los cónyuges), lo más habitual es convivir con la madre, siendo escasa la proporción de hogares en los que los hijos tienden a residir solo con el padre. La existencia de un modelo de hogar monoparental donde se convive con la madre es clara en todas las regiones analizadas ${ }^{6}$, si bien el nivel que muestra esta opción es especialmente bajo en el caso de los países asiáticos, donde el modelo tradicional de convivencia con ambos padres es claramente predominante.

La conjunción de estos elementos junto a las anteriores tendencias de descenso del peso de niños y jóvenes entre la población total ayuda a concebir escenarios en los que cada vez será más habitual ver a niños conviviendo solo con sus progenitores (o con la madre, en su caso), con una menor disposición de otros individuos de su misma edad y características demográficas en su entorno de referencia. Esto, probablemente, tendrá una clara influencia sobre los modos y procesos que concurren en la conformación de las relaciones interpersonales para los niños y los adolescentes y sobre su proceso de socialización.

\section{La nupcialidad adolescente}

Las primeras etapas de la vida del ser humano suelen tener características y rasgos particulares que las distancian claramente del modo de vida de los adultos. De hecho, para muchas culturas son los comportamientos que definen ese modo de vida adulta los que determinan el fin de la infancia y la adolescencia y el paso de cada individuo a un estadio diferente en su evolución como persona y ciudadano. Sin embargo, no faltan ocasiones en los que la vida adulta se inmiscuye en la vida adolescente, forzando ese tránsito que, en la mayoría de las veces, supone un drástico cambio para sus protagonistas. El matrimonio adolescente constituye uno de esos procesos no exentos de consecuencias, a menudo totalmente indeseadas, en los que los jóvenes se ven forzados a adoptar pautas de comportamiento propios de la vida adulta. Si a esto le añadimos la estrecha relación de este fenómeno con los problemas que tienen que ver con el logro de una determinada salud sexual y reproductiva, puede entenderse que los organismos internacionales y los gobiernos responsables procuren prestar una especial atención a la incidencia de estos fenómenos y a sus repercusiones desde hace bastantes años (Lassonde, 1997; UNICEF, 2014).

En la actualidad, el matrimonio o nupcialidad adolescente sigue siendo un fenómeno bien presente en muchas sociedades del planeta. Ningún país se ve totalmente exento de cierta proporción de uniones matrimoniales en edades típicas de la adolescencia, pero las diferencias entre unas regiones y otras son importantes todavía.

En este caso, los datos disponibles nos obligan a considerar, para poder capturar una buena parte de este fenómeno, los matrimonios acontecidos en el grupo de edad de 15 a 19 años y, con suerte, cuando hay datos para ello, las uniones celebradas con personas menores de 18 años.

La situación en el África Subsahariana y en algún país asiático y caribeño destaca claramente en el mapa elaborado. Así, más de un 20\% del total de mujeres casadas en las edades típicamente reproductivas (15 a 49 años) acceden al matrimonio entre los 15 y los 19 años. La situación mejora un poco en otras zonas como el sur de Asia y, fundamentalmente América Latina y el Caribe, pero la proporción sigue siendo relevante, situándose entre un $10 \%$ y un $20 \%$. Es, a todas luces, un fenómeno donde sigue siendo necesaria una clara intervención y la revisión urgente de las políticas sociales y educativas sobre la adolescencia.

Mapa 4. Porcentaje de mujeres casadas entre 15 y 19 años respecto al total de mujeres en edad reproductiva (15-49 años). Media del período 2012 a 2018.

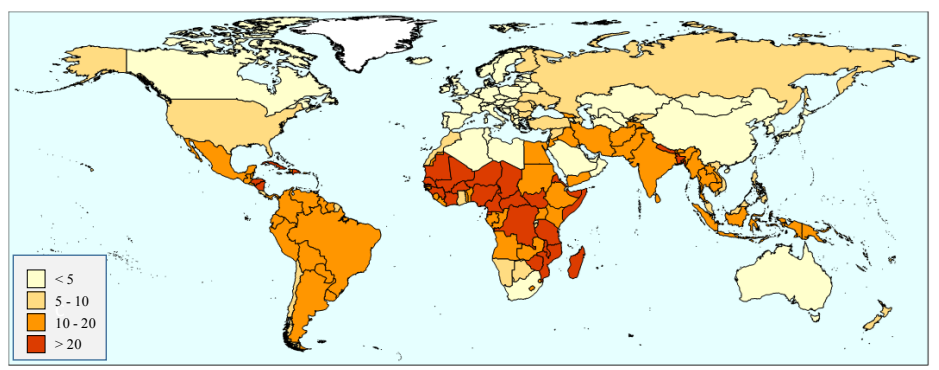

Elaboración propia a partir de los datos de: United Nations, Department of Economic and Social Affairs, Population Division (2018b). Estimates and projections of women of reproductive age who are married or in a union: 2018 Revision.

New York, (https://www.un.org/en/development/desa/population/index.asp).

\footnotetext{
Pese a la carencia de información específica a nivel mundial, hay que hacer constar que en muchos países esta corresidencia con ambos padres o con un solo progenitor no excluye la situación de corresidencia multigeneracional donde se convive, además, con abuelos.

6 Debe tenerse en cuenta que en muchos países la legislación existente otorga la custodia de los hijos a la madre en caso de separación o divorcio.
} 
Una buena noticia al respecto, aunque solo en parte, es que los datos disponibles muestran una clara evolución descendente del fenómeno. Si apreciamos los datos representados en el gráfico 5 (panel A) podemos ver cómo, además de vislumbrar la posición de las diferentes regiones del mundo con respecto al matrimonio adolescente (destacando la alta incidencia en países de África y Asia), el hecho de que casi todos los puntos se sitúen por encima de la línea de igualdad y se acerquen a los valores del eje para el período 1970-76 (en vertical) supone que, para 2012-18, hay una menor incidencia de este tipo de nupcialidad. Lo negativo de la evolución mostrada es que algunos países han aumentado su peso y, también, que una buena parte de los puntos que representan los países de América Latina se sitúan muy cercanos a la línea de igualdad, lo que significa que ha habido cierta tendencia a la caída de este tipo de matrimonios en esta región, pero no ha sido excesivamente importante. La falta de información clara sobre el fenómeno no deja apreciar toda su dimensión, donde habría que incluir la incidencia de la cohabitación en varios países latinoamericanos (Esteve et al., 2012). El claro contraejemplo en estas dinámicas de nupcialidad adolescente es el caso de Europa, que muestra una clara agrupación junto al eje vertical y en valores bajos, mostrando importantes avances en la reducción de este fenómeno.

Gráfico 5. Características de la nupcialidad adolescente en el mundo: A) Cambio temporal del porcentaje de mujeres casadas de 15-19 años entre 1970-76 y 2012-18 en el mundo. B) Relación entre los sexos en personas casadas antes de los 18 años (sobre el porcentaje de personas de 20-24 años) en el mundo. Datos para el período 2012-2018.
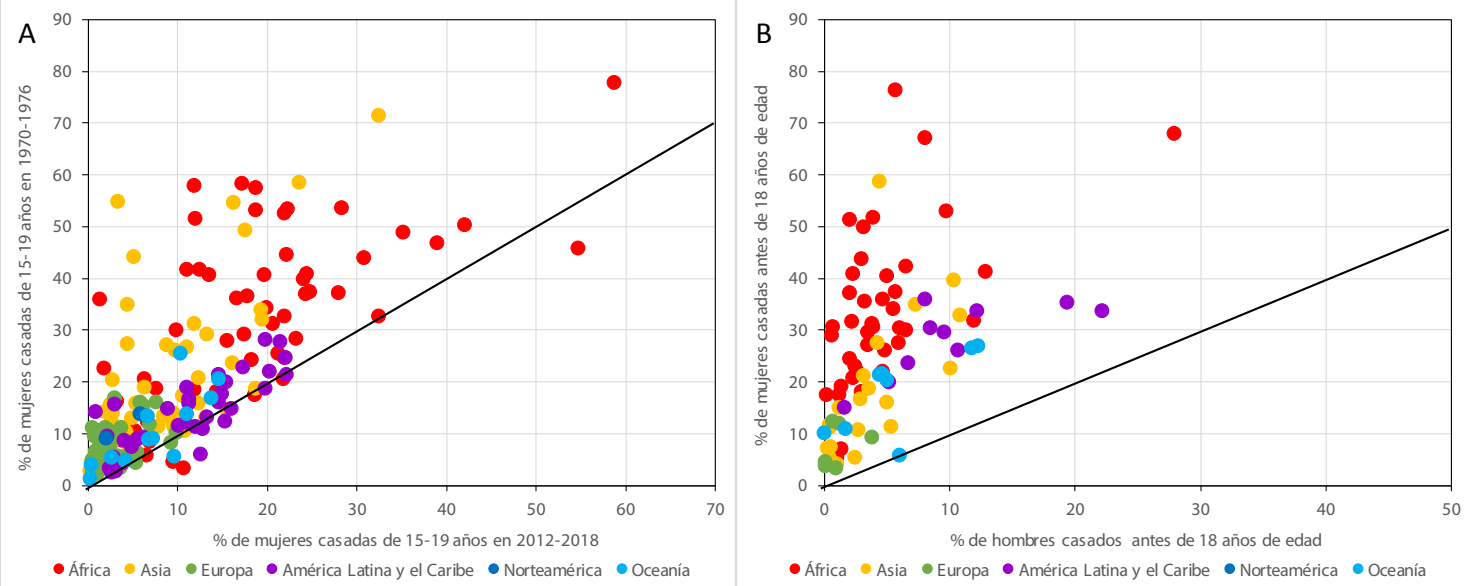

Elaboración propia a partir de los datos de: United Nations,

Department of Economic and Social Affairs, Population Division (2018b).

Estimates and projections of women of reproductive age who are married or in a union: 2018 Revision. New York, (https://https://www.un.org/en/development/desa/population/index.asp) y UNICEF (2019). UNICEF global databases, 2019, based on Demographic and Health Surveys (DHS), Multiple Indicator Cluster Surveys (MICS) and other nationally representative surveys, (https://data.unicef.org).

En el panel B del gráfico 5, se puede apreciar cómo la incidencia de la nupcialidad adolescente afecta fundamentalmente al grupo de mujeres, mientras que la proporción de varones afectados por el matrimonio temprano es claramente menor. Nuevamente, África y Asia aparecen con una clara feminización de este tipo de uniones, mientras que América Latina muestra la situación de algunos países donde, si bien las mujeres siguen siendo las principales protagonistas, los varones experimentan valores relevantes en el conjunto de puntos que representan los países con datos disponibles.

\section{La fecundidad adolescente}

Buena parte de los matrimonios adolescentes conllevan asociada la experiencia de fecundidad de sus protagonistas. Así, la relación entre matrimonio y fecundidad adolescente es bastante considerable (gráfico 6), con un índice de correlación de 0,65 y una alta incidencia, fundamentalmente entre países del África Subsahariana y de América Latina. Asia también presenta una alta incidencia de este fenómeno, pero es aventajada por las dos regiones anteriores. 
Gráfico 6. Relación entre el matrimonio temprano (\%) y la tasa de fecundidad adolescente (mujeres de 15-19 años) en el mundo.

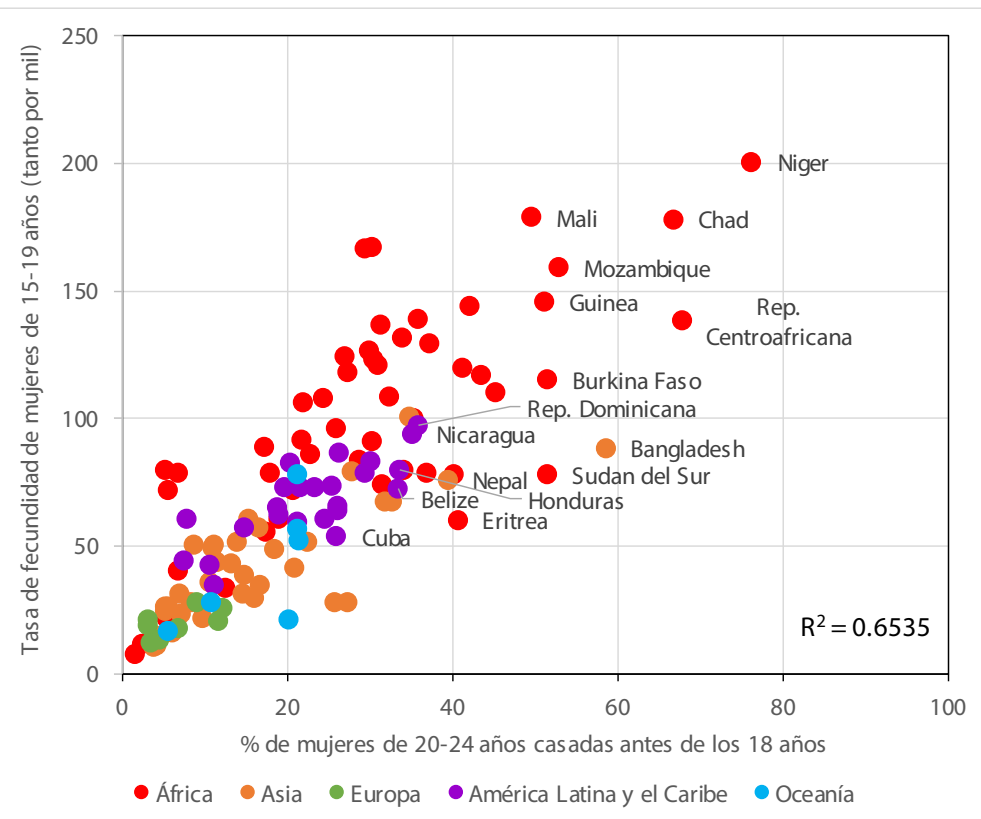

Elaboración propia a partir de: Tasas de Fecundidad: United Nations, Department of Economic and Social Affairs, Population Division (2019b). World Population Prospects 2019, (https://population.un.org/wpp/). \% de Mujeres Casadas antes de los 18 años: UNICEF (2019). UNICEF global databases, 2019, based on Demographic and Health Surveys (DHS), Multiple Indicator Cluster Surveys (MICS) and other nationally representative surveys, (https://data.unicef.org).

Solo hay datos disponibles para 123 países.

La evolución de esta fecundidad adolescente (medida como la tasa específica de fecundidad en mujeres de 15 a 19 años) es de una clara caída desde que existen series de datos para los diversos países y regiones del mundo (gráfico 7 , panel A), aunque las diferencias en esta evolución denotan que todavía se puede avanzar bastante, especialmente en dos regiones: África (fundamentalmente la región Subsahariana) y América Latina y el Caribe. De hecho, si consideramos el peso de la fecundidad de las jóvenes de 15 a 19 años, América Latina se destaca como la región del mundo con una mayor proporción de su fecundidad dentro de este grupo de edad (en torno a un 15\% - gráfico 7, panel B). Su situación es, además, bastante diferente con respecto a otras zonas: África se sitúa en torno al $10 \%$ y el resto de las regiones se ubican por debajo del $6 \%$.

Gráfico 7. La evolución de la fecundidad adolescente (15-19 años) en el mundo: A) Evolución y perspectivas de las tasas de fecundidad de mujeres de 15-19 años. B) Evolución del peso relativo (\%) de la fecundidad de 15-19 años en la fecundidad total de 15-49 años.
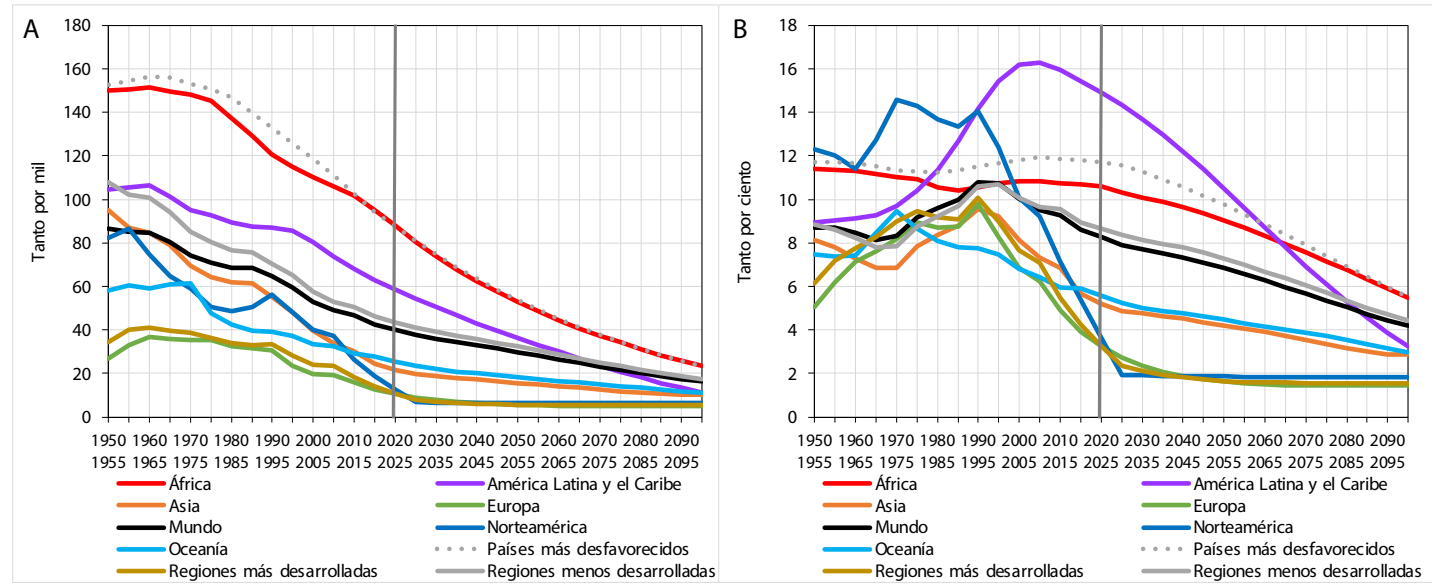

Elaboración propia a partir de los datos de: United Nations, Department of Economic and Social Affairs, Population Division (2019b). World Population Prospects 2019, (https://population.un.org/wpp/).

Esta particular situación de América Latina y el Caribe está siendo desde hace años objeto de un particular seguimiento y atención, dado que el fenómeno muestra niveles preocupantes para asegurar un buen estado de bienestar 
sexual y reproductiva (Rodríguez Vignoli, 2014; Rodríguez Vignoli, 2017) y, además, presenta comportamientos específicos según el nivel educativo de las mujeres que requieren de atención concreta para una correcta intervención (Esteve Palós y Florez-Paredes, 2014; UNICEF, 2014).

\section{La mortalidad y sus causas en la infancia y la adolescencia}

Al tratar sobre el bienestar de niños y adolescentes, los indicadores sobre mortalidad son la evidencia más directa que podemos tener de cómo se encuentra la salud de los niños y los jóvenes. De hecho, la tasa de mortalidad infantil suele utilizarse habitualmente para valorar el grado de desarrollo de una determinada población.

Conviene advertir al lector que las referencias a la edad para analizar el fenómeno de la mortalidad son variadas y así, por ejemplo, no es lo mismo hablar de la mortalidad infantil desde una perspectiva demográfica, que solo cuenta las defunciones de niños antes de su primer aniversario (0 años) que de la mortalidad en la infancia, que puede incluir a los menores de 5 años o bien otros grupos de edad. No es raro hablar de tasas de mortalidad juvenil para referirse a edades tan variadas como los 5 a 9 o de 10 a 14 años. Se sugiere que, en cada caso, se tenga muy en cuenta cuáles son las edades de referencia para evitar confusiones acerca de los niveles detectados y las situaciones que se pretende analizar.

El indicador de la tasa de mortalidad infantil (gráfico 8) nos da una clara idea de cómo ha mejorado la situación de la salud de los niños en la segunda mitad del siglo XX y hasta la actualidad y de los buenos pronósticos que se hacen para el siglo XXI, si bien, todavía para 2050 habrá diferencias reseñables entre unas regiones y otras.

Gráfico 8. Evolución y perspectivas de la mortalidad infantil (0 años) mundial.

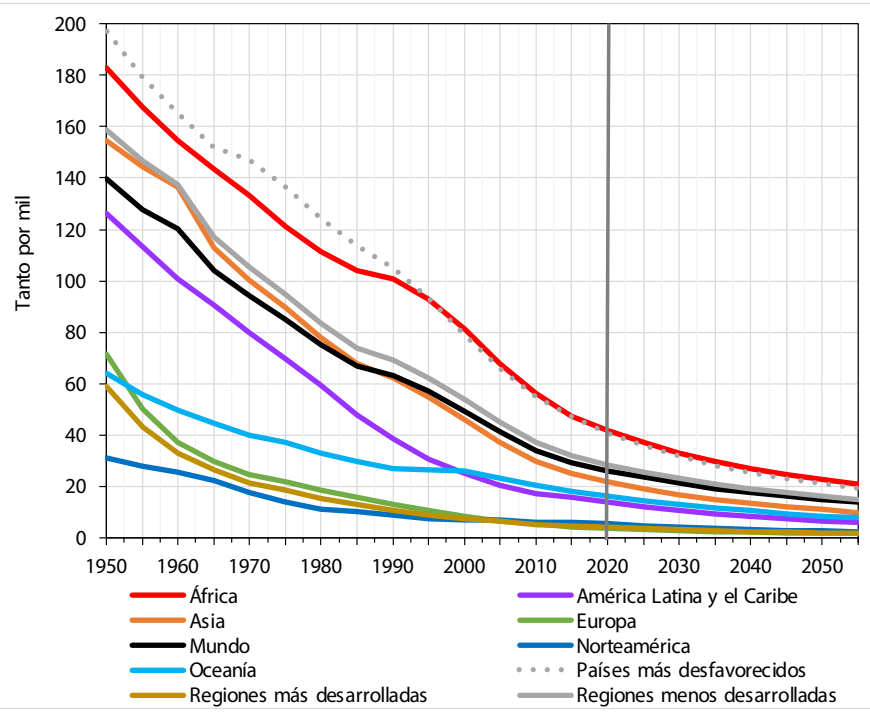

Elaboración propia a partir de los datos de: United Nations, Department of Economic and Social Affairs, Population Division (2019b). World Population Prospects 2019, (https://population.un.org/wpp/).

En 1950, el mundo presenta enormes disparidades con respecto a la salud de los niños. Mientras Europa y Norteamérica ya habían experimentado caídas sustantivas de esta mortalidad, América Latina, Asia y, sobre todo África, mostraban aún niveles propios de épocas del pasado, cuando la vida de un niño recién nacido era uno de los elementos más frágiles de los sistemas demográficos (Pérez Moreda et al., 2015). Conviene destacar que en la segunda mitad del siglo XX y hasta hoy, se ha conocido una rápida reducción de la incidencia de este tipo de mortalidad y que los países con niveles bajos aún han mejorado más su situación. De hecho, la mayor parte de los países desarrollados tienen tasas de mortalidad situadas entre 3 y 6 fallecidos por cada mil nacidos vivos. En el mundo en desarrollo, la evolución también ha sido favorable, aunque aún persisten diferencias importantes y los países africanos, por ejemplo, todavía experimentan una mortalidad entre 10 y 13 veces mayor que los países más desarrollados.

Si examinamos el peso de la mortalidad en otras edades de la infancia y la adolescencia (gráfico 9) podemos ver como la mortalidad es especialmente alta cuanto más cerca nos encontramos del momento del nacimiento. De hecho, pese a que en algunas regiones supone un peso más elevado (como en Asia), la mortalidad infantil supone casi la mitad de las defunciones ocurridas entre 0 y 17 años. La especial vulnerabilidad y sensibilidad del ser humano a las condiciones del embarazo, del parto y del entorno ayudan a explicar este mayor peso de los fallecimientos en los niños de 0 años. La experiencia de los países con un mayor nivel de desarrollo ha demostrado que la mortalidad disminuye de forma clara y contundente según aumenta la edad hasta la adolescencia. Así, si atendemos tanto al valor de las probabilidades de muerte por edad (indicador de intensidad del fenómeno) como a su peso relativo entre el total de defunciones de 0 a 
17 años, podemos ver que los mayores niveles de mortalidad tras la ocurrida a 0 años, se da en los niños de 1 a 4 años, después se reduce mucho en los dos siguientes grupos de edad (5 a 9 y 10 a 14 años) y vuelve a elevarse claramente en el grupo adolescente de 15 a 19 años. De una forma más concreta, en las regiones con mayor nivel de desarrollo la mayor parte de las muertes se dan en los grupos extremos, es decir, a los 0 años y de 15 a 19 años. En las regiones menos desarrolladas, la mortalidad de 1 a 4 años sigue siendo importante en la distribución del fenómeno y este hecho pone de relieve la existencia de patrones de causa de muerte distintos en unas poblaciones y otras.

Gráfico 9. Niveles y peso (\%) de la mortalidad en la infancia y adolescencia por edades y regiones.

Quinquenios 2015-20 y 2050-55.
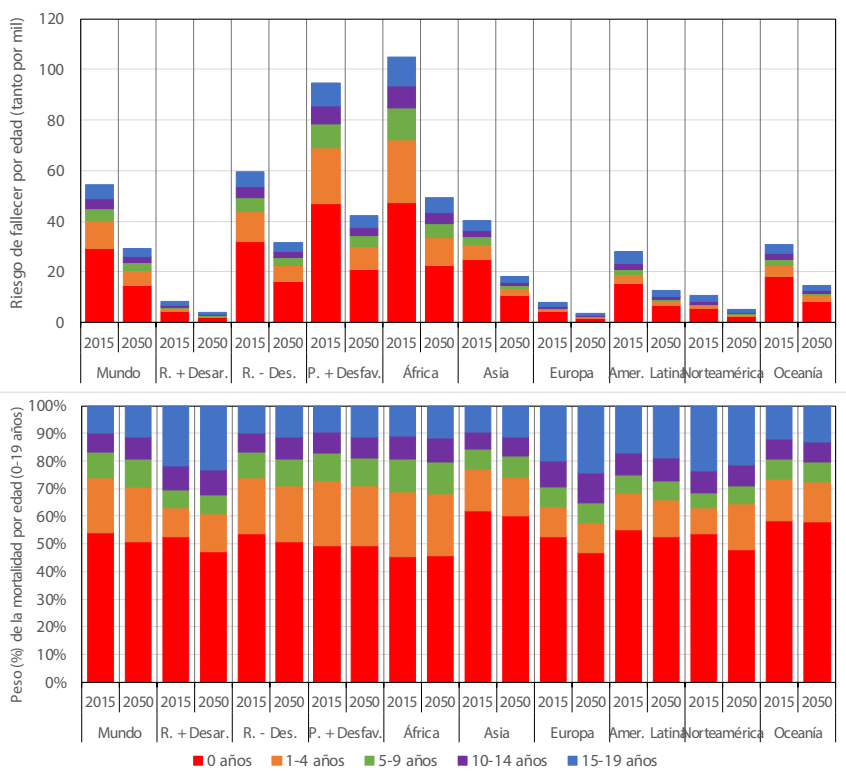

Elaboración propia a partir de los datos de: United Nations, Department of Economic and Social Affairs, Population Division (2019b). World Population Prospects 2019, (https://population.un.org/wpp/).

El análisis de las causas de muerte en la infancia y la adolescencia refleja la influencia de factores claramente diferentes según la edad de los fallecidos. Durante la primera infancia (0 a 4 años - gráfico 10) hay que distinguir las causas que afectan a los niños que fallecen en torno al nacimiento (mortalidad neonatal - primer mes de vida) de las que ocurren en los siguientes años hasta cumplir los 4. La mortalidad neonatal se caracteriza por un elevado peso de los problemas relacionados con la prematuridad, las complicaciones durante el parto y las causas congénitas (de carácter perinatal), cuyo peso conjunto supone aproximadamente un $70 \%$ de los fallecimientos a esta edad. Entre los niños con otras edades hasta los 4 años, se sigue apreciando el peso de estos factores, pero a ellos se suma la incidencia de las enfermedades infecciosas (diarreas e infecciones respiratorias, principalmente). Si se compara el perfil de los países ricos con el de los países pobres, cabe apreciar la mayor incidencia en estos últimos de este patrón de mortalidad infeccioso, donde junto a las causas perinatales y congénitas antes mencionadas, hay que añadir el peso de la malaria, la meningoencefalitis, la tosferina, el sarampión y el VIH-SIDA. Muchas de estas enfermedades infecciosas apenas provocan fallecimientos en los países más desarrollados y su incidencia en el patrón de mortalidad es realmente reducida y, en algunos casos, anecdótica.

Gráfico 10. La mortalidad de los niños de 0 a 4 años por causas de muerte (\%) en el mundo. 2017.

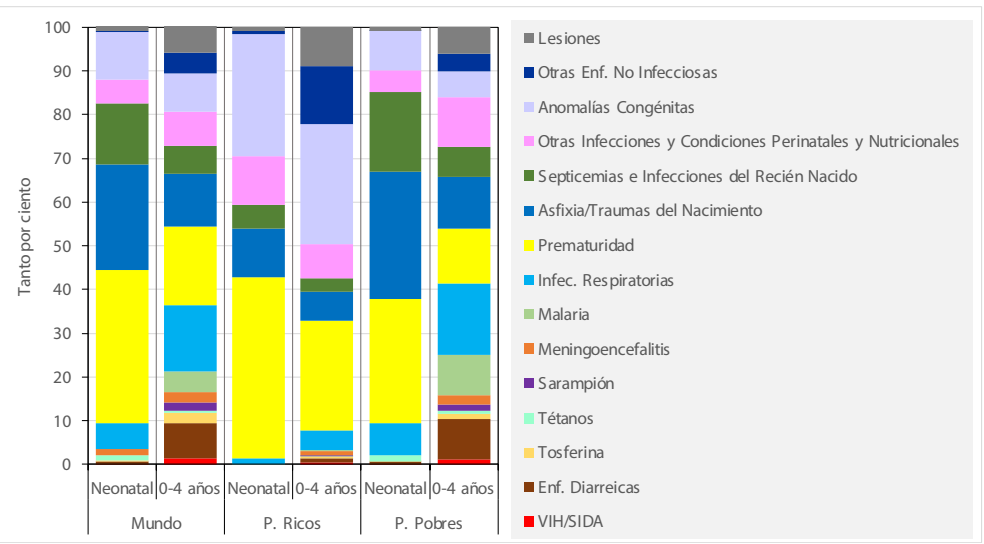

Elaboración propia a partir de los datos de: World Health Organization (2020). The Global Health Observatory, (https:// www.who.int/data/gho). 
A medida que los individuos crecen, los riesgos y causas que pueden explicar su nivel de salud (en este caso, su mortalidad) cambian de forma importante. Su vulnerabilidad se hace menor en la medida que aumenta su resistencia fisiológica y su sistema inmunitario se hace cada vez más capaz y potente. Es por ello, que lo esperable es un descenso claro de la mortalidad con el crecimiento. Sin embargo, la entrada en juego de otros factores de carácter social y psicológico, relacionados en buena medida con los hábitos, el entorno y los comportamientos, ayuda a entender porque en ciertos contextos la mortalidad de los adolescentes vuelve a elevarse con respecto a las edades precedentes.

Gráfico 11. La mortalidad de los adolescentes (10-19 años) por causas de muerte (\%) en el mundo. 2016.
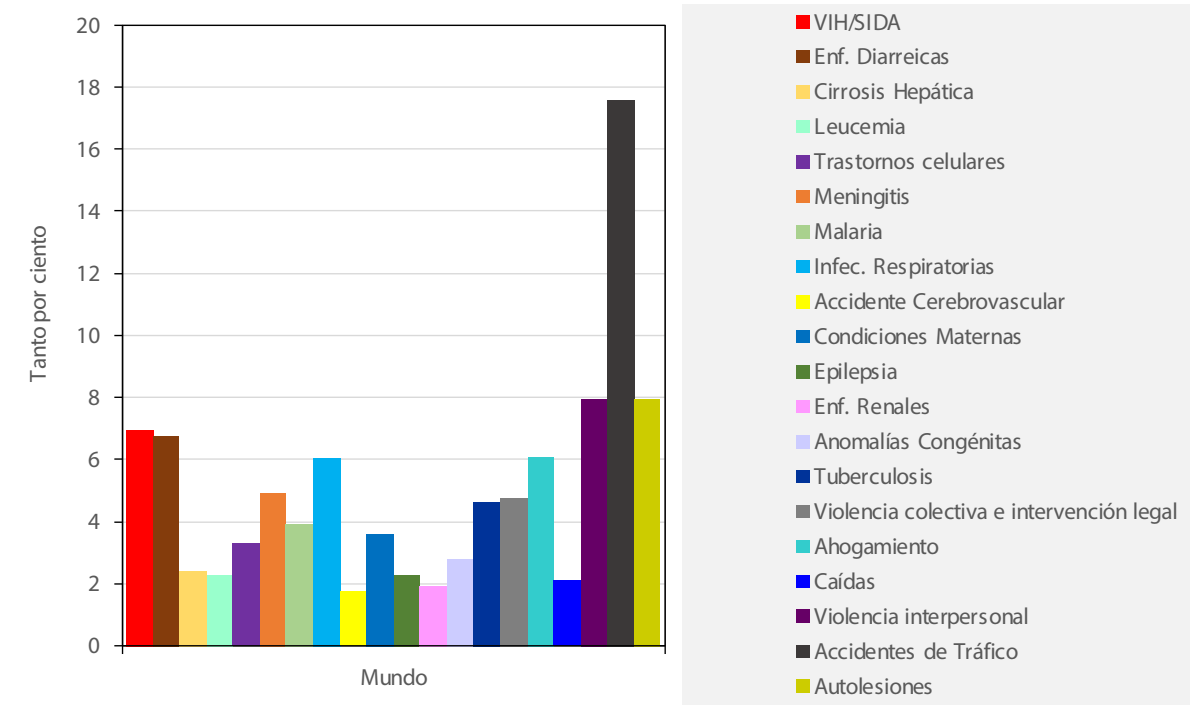

Elaboración propia a partir de los datos de: World Health Organization (2020). The Global Health Observatory, (https:// www.who.int/data/mncah).

Si bien existen datos sobre las causas de mortalidad adolescente para muchos países, no hemos podido localizar una serie que se distribuya de una manera similar a la planteada en este trabajo, por lo que nuestro análisis sobre este fenómeno se referirá únicamente al patrón que se aprecia para este grupo de edad en el mundo (gráfico 11). Si tratamos de agrupar las causas por su naturaleza infecciosa o no infecciosa y por el tipo de patologías existentes, podríamos resumir que, a nivel mundial, un 10\% de las muertes de adolescentes tienen que ver con problemas congénito-hereditarios, un $44 \%$ estarían relacionadas con enfermedades de tipo infeccioso y un $46 \%$ con causas relacionadas con violencia, traumatismos y accidentes. De hecho, en el mundo, la principal causa de muerte entre individuos de 10 a 19 años son los accidentes de tráfico, cuyo peso sería solo ligeramente inferior a lo que supondría la suma de las lesiones relacionadas con los distintos tipos de violencia (incluyendo las autolesiones). Este patrón de causas de muerte pone de manifiesto la especial dificultad que supone la lucha contra este tipo de mortalidad en ciertos lugares del mundo, pues no se trata solo de conseguir contener la propagación de ciertas enfermedades infecciosas, sino también, de influir en los comportamientos y actitudes de los individuos y de los gobiernos y grupos de interés.

\section{El papel de los niños y los adolescentes en las migraciones internacionales}

Por último, no podemos terminar esta revisión demográfica de la situación de la infancia y la adolescencia en el mundo sin prestar atención a su papel dentro de los procesos migratorios internacionales. Teniendo en cuenta el volumen de población que representan, las migraciones entre países son de menor cuantía que los movimientos realizados dentro de las fronteras de un determinado país. De hecho, las migraciones internacionales, cuyo registro no siempre goza de la fiabilidad deseable, afectan entre un 3.5\% y un 4\% de la población mundial. Dentro de este contingente, los niños y los adolescentes suponen un 14\% aproximadamente (entre 31 y 39 millones de individuos según la fuente (Bhabha and Abel, 2020) y, teniendo en cuenta las áreas de destino en las que han sido detectados, África, América Latina y el Caribe y Asia son las regiones del planeta que implican una mayor proporción de estos migrantes (gráfico 12). 
Gráfico 12. Distribución (\%) por edad de los niños y adolescentes en

las migraciones internacionales en el mundo. Año 2019.

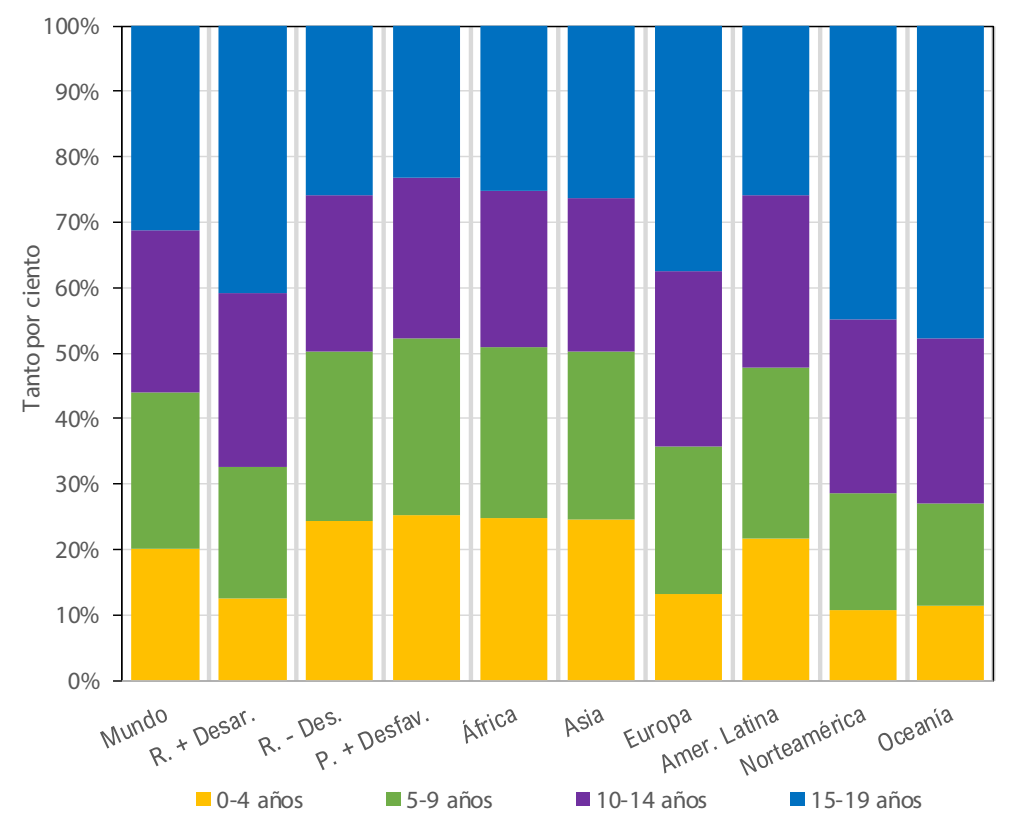

Elaboración propia a partir de los datos de: United Nations, Department of Economic and Social Affairs, Population

Division (2019a). International Migrant Stock 2019 (United Nations database, POP/DB/MIG/Stock/Rev.2019).

Si bien las disparidades económicas suponen la principal causa de los movimientos migratorios, no faltan otros motivos que, en muchas ocasiones, suponen una mayor vulnerabilidad y riesgo para sus protagonistas. Así, las migraciones de los desplazados que buscan refugio en otro país por razones de violencia, conflicto o persecución, se han convertido en una parte nada desdeñable del monto total de movimientos migratorios, llegando a suponer el $8 \%$ de todos los desplazamientos entre países registrados en 2017. Mientras las migraciones económicas se componen fundamentalmente de personas en edad laboral (20 a 34 años), las migraciones de los desplazados-refugiados tienen una elevada participación de niños y adolescentes, que suponen el 50\% del total de personas en esta situación. Entre los refugiados, los niños y los adolescentes destacan en el continente africano (suponen más de la mitad del contingente) y, también, en Asia, en América Latina y Norteamérica (incluyendo aquí Centroamérica).

La distribución por sexos, que puede hallarse muy sesgada por la influencia del distinto registro de varones y mujeres, revela un predominio claramente masculino en prácticamente todas las regiones, especialmente en el caso de Asia, donde las migraciones femeninas en la infancia y la adolescencia son algo menores que las de los varones. Por el contrario, entre las migraciones feminizadas destaca el continente africano, donde la razón entre los sexos es de unos 96 varones por cada 100 mujeres migrantes. Estos resultados contrastan con los de las estadísticas migratorias generales sobre África (que muestran una mayor proporción de varones) y deben ser tomados con cierta cautela, porque la confección de estas estadísticas no está exenta de posibles sesgos. Sin embargo, cabe apuntar como posible razón de esta peculiar situación del continente africano, el hecho de que buena parte de la población más joven en movimiento esté constituida por mujeres, niñas y adolescentes, que conforman una parte sustantiva de los movimientos de refugiados en este continente, especialmente en el África Subsahariana (Zlotnik, 2003).

\section{Conclusiones}

Siguiendo un propósito inicial que jugaba con un doble objetivo: 1) examinar la situación de la infancia y la adolescencia actual y 2) mostrar las posibilidades metodológicas que los indicadores demográficos tienen para ello, en este trabajo hemos podido echar un vistazo general a los principales rasgos que caracterizan a la población infantil y juvenil en nuestros días. En este sentido, es evidente que el uso de indicadores demográficos es necesario para ahondar en nuestro conocimiento de varios de los fenómenos que afectan a este grupo de población en la actualidad. Muchos de ellos son de tal calado y requieren tal urgencia, que deberían conformar la agenda de muchos de los programas de acción en los próximos años. Sin duda, no solo las cuestiones relativas a la salud individual, sino también otras relacionadas con la salud sexual y reproductiva, que afectan a los comportamientos y las relaciones con otras personas, precisan de una clara atención desde las diversas disciplinas y especialidades encargadas de su estudio y de la propuesta de soluciones.

Los rasgos cuantitativos de la infancia y adolescencia no terminan en la magnitud o entidad de unas determinadas cifras. Son una herramienta y una posibilidad para ahondar en diversos fenómenos y procesos, que no se agotan con 
un simple recuento de casos. Las implicaciones de la disminución del tamaño de los hogares y de cómo se vertebran las estructuras familiares y de corresidencia son un buen ejemplo de ello, entre otros aspectos. La disponibilidad de pares para los niños y los adolescentes en los próximos años va a seguir un proceso de reducción que, en algunos contextos, tiene importantes implicaciones para el modo y calidad de vida de los mismos. Téngase en cuenta, sobre este particular, el distinto tipo de relaciones sociales que pueden desarrollar los niños y los adolescentes de los ámbitos urbano y rural en muchas zonas de Europa donde las poblaciones decrecen. Este trabajo ha mostrado, en este sentido, varios aspectos que requieren de un mayor esfuerzo de estudio y análisis, sobre todo en ámbitos geográficos y territoriales más concretos.

Los datos e indicadores demográficos permiten adoptar un enfoque claramente centrado en los niños y los adolescentes como objeto y sujeto de estudio y cuando esto no resulta directamente posible, el investigador tiene a su disposición herramientas metodológicas y fuentes de información como censos y encuestas, que permiten realizar un análisis de los fenómenos, manteniendo sobre este grupo su centro focal de su estudio. Es tarea del investigador aprender a manejar y utilizar bien estas herramientas y, sobre todo, a extraer de los datos las interpretaciones más certeras posibles, que permitan una buena identificación, diagnóstico y examen de los factores que concurren en el devenir de un determinado fenómeno. Sin duda, la complementariedad con otras disciplinas acabará constituyendo la base para el diseño de un plan efectivo de acción.

Este trabajo está lejos de ofrecer los procedimientos necesarios para conseguir este tipo de avances en la investigación, pero ha puesto de relieve cómo se puede partir de datos disponibles para avanzar en nuestro conocimiento y comprensión de algunos de los procesos sustantivos que afectan a la infancia y a la adolescencia y para al menos, dar un primer paso en su solución.

\section{Referencias bibliográficas}

Arango Vila-Belda, J. (2004). La población mundial. En J. Romero González (ed.), Geografía Humana. Procesos, riesgos e incertidumbres en un mundo globalizado (pp. 55-99). Barcelona: Ariel.

Arnold, F., Kishor, S., Roy, T.K. (2002). Sex-selective abortions in India. Population and Development Review, 28, $759-785$.

Arnold, F., Zhaoxiang, L. (1986). Sex preference, fertility, and family planning in China. Population and Development Review, 12-Junio(2), 221-246.

Arokiasamy, P. (2002). Gender preference, contraceptive use and fertility in India: regional and development influences. International Journal of Population Geography, 8(1), 49-67.

Attané, I. (2006). The demographic impact of a female deficit in China, 2000-2050. Population and Development Review, 32(4), 755-770.

Attané, I. (2013). A geography of discrimination. En I. Attané (ed.), The demographic masculinization of China: Hoping for a son (pp. 35-50). Heidelberg: Springer International Publishing.

Bhabha, J., Abel, G. (2020). Los niños y la migración insegura. En Migraciones, Organización Internacional para las Migraciones ed. Informe sobre las Migraciones en el Mundo 2020 (pp. 251-281). Ginebra: Organización Internacional para las Migraciones.

Chesnais, J. C. (1992). The demographic transition. Stages, patterns and economic implications. Oxford: Clarendon Press Oxford.

Das Gupta, M., Zhenghua, J., Bohua, L., Zhenming, X., Chung, W., Hwa-Ok, B. (2002). Why is son preference so persistent in East and South Asia? A cross-country study of China, India, and the Republic of Korea. Policy Research Working Paper World Bank, 2942.

Esteve Palós, A., Lesthaeghe, R., López-Gay, A. (2012). The Latin American cohabitation boom, 1970-2007. Population and Development Review, 38(1), 55-81.

Esteve Palós, A., Florez-Paredes, E. (2014). Edad a la primera unión y al primer hijo en América Latina: estabilidad en cohortes más educadas. Notas de Población, 41(99), 39-65.

Goodkind, D. (1996). On substituting sex preference strategies in East Asia: Does prenatal sex selection reduce postnatal discrimination? Population and Development Review, 22(1), 111-125.

Hesketh, T., Lu, L., Xing, Z. W. (2011). The consequences of son preference and sex-selective abortion in China and other Asian countries. CMAJ: Canadian Medical Association Journal, 183(12), 1374-1377.

Lassonde, L. (1997). Los desafios de la demografía. ¿Qué calidad de vida habrá en el siglo XXI? México: Universidad Nacional Autónoma de México, Fondo de Cultura Económica.

Lesthaeghe, R., Van de Kaa, D. J. (1986) . Two demographic transitions? En D.J. Van de Kaa, R. Lesthaeghe (eds.), Population: Growth and decline (pp. 9-24). Deventer: Van Loghum Slaterus.

Livi-Bacci, M. (1993). Introducción a la demografia. Barcelona: Ariel.

Livi-Bacci, M. (2002). Historia mínima de la población mundial. Barcelona: Ariel.

Notestein, F.W. (1945). Population: the long view. En T.W. Schultz (ed.), Food for the world (pp. 36-57). Chicago: Chicago University Press.

Pérez Moreda, V., Reher, D.-S., Sanz Gimeno, A. (2015). La conquista de la salud. Mortalidad y modernización en la España contemporánea. Madrid: Marcial Pons.

Reher, D.-S. (2004). The demographic transition revisited as a global process. Population, Space and Place, (10), 19-41. 
Rodríguez Vignoli, J. (2014) Fecundidad adolescente en América Latina: una actualización. En Asociación Latinoamericana de Población (ALAP), ed., Comportamiento reproductivo y fecundidad en América Latina: una agenda inconclusa (pp. 33-65). Río de Janeiro: Asociación Latinoamericana de Población (ALAP).

Rodríguez Vignoli, J. (2017) Fecundidad no deseada entre las adolescentes latinoamericanas. Un aumento que desafia la salud sexual y reproductiva y el ejercicio de derechos. Santiago de Chile: CEPAL- UNFPA.

Svedberd, P. (1990). Undernutrition in Sub-Saharan Africa: Is there a gender bias? The Journal of Development Studies, 26(3), 469-486.

UNICEF (2014). El estado mundial de la infancia en 2014 en cifras. Todos los niños y niñas cuentan. New York: Fondo de las Naciones Unidas para la Infancia (en línea). https:/www.unicef.org/spanish/sowc/.

UNICEF (2018). UNICEF global databases - Child migrants and refugees (international migrants, refugees, asylum seekers and internally displaced persons) - December 2018, (en línea). https://data.unicef.org.

UNICEF (2019). UNICEF global databases, 2019, based on Demographic and Health Surveys (DHS), Multiple Indicator Cluster Surveys (MICS) and other nationally representative surveys, (en línea). https://data.unicef.org.

United Nations - Department of Economic and Social Affairs (2017). Household size and composition around the world 2017. Data booklet. New York: United Nations.

United Nations - Department of Economic and Social Affairs (2018a). Database on Household Size and Composition 2018, (en línea) https://population.un.org/Household/index.html.

United Nations - Department of Economic and Social Affairs (2018b). Estimates and projections of women of reproductive age who are married or in a union: 2018 Revision, (en línea). https:/www.un.org/en/development/desa/population/index.asp.

United Nations - Department of Economic and Social Affairs (2019a). International Migrant Stock 2019 (en línea) https://www. un.org/development/desa/en/news/population/international-migrant-stock-2019.html

United Nations - Department of Economic and Social Affairs (2019b). World Population Prospects 2019, (en línea). https://population.un.org/wpp/.

van Bavel, J., Reher, D. (2013). The baby boom and its causes: What we know and what we need to know. Population and Development Review, 39(2), 257-288.

World Health Organization (2020). The Global Health Observatory (en línea). https://www.who.int/data/gho.

Zlotnik, H. (2003). The global dimensiones of female migration. Migration Information Source, March(1) (en línea). https:// www.migrationpolicy.org/article/global-dimensions-female-migration/. 